\title{
Toque no bebê hospitalizado sob a luz da teoria de Martha Elizabeth Rogers: reflexões de enfermagem
}

\author{
Touch the hospitalized baby under the light of Martha's Theory Elizabeth Rogers: nursing \\ reflections \\ Tocar al bebé hospitalizado bajo la luz de la teoría de Martha Elizabeth Rogers: reflexiones de \\ enfermería
}

Recebido: 26/07/2021 | Revisado: 03/08/2021 | Aceito: 07/08/2021 | Publicado: 14/08/2021

Isis Ferraz Barbosa

ORCID: https://orcid.org/0000-0002-2171-4104

Universidade Federal de Mato Grosso do Sul, Brasil E-mail: isis.barbosa@ufms.br

Vanessa Giavarotti Taboza Flores

ORCID: https://orcid.org/0000-0002-1396-5843

Universidade Federal de Mato Grosso do Sul, Brasil E-mail: vanessa.flores@ufms.br

Adriano Menis Ferreira

ORCID: https://orcid.org/0000-0002-4054-768X

Universidade Federal de Mato Grosso do Sul, Brasil E-mail: adriano.ferreira@ufms.br

Marcos Antônio Ferreira Júnior

ORCID: https://orcid.org/0000-0002-9123-232X

Universidade Federal de Mato Grosso do Sul, Brasil E-mail: marcos.junior@ufms.br Verusca Soares de Souza

ORCID: https://orcid.org/0000-0002-4054-768X Universidade Federal de Mato Grosso do Sul, Brasil E-mail: verusca.soares@ufms.br

\begin{abstract}
Resumo
Objetivo: Promover reflexões acerca do cuidado integral de enfermagem sob a perspectiva da Teoria de Martha Elizabeth Rogers e o contato dos bebês com suas mães e a equipe de enfermagem. Metodologia: Trata-se de artigo de análise teórico-reflexiva sustentada pelo referencial teórico da teoria de Martha Elizabeth Rogers, para o qual foi realizada uma busca por produções que versassem a respeito do tema na plataforma Google Acadêmico e no Pubmed, baseados na vivência de duas enfermeiras com experiência em cuidados neonatais, que ocorreu nos meses de junho e julho de 2021. Resultados: Os três eixos foram detalhados, aliando as contribuições da literatura, com as possibilidades de aplicação do arcabouço teórico. Na prática assistencial em neonatologia, o toque beneficia o desenvolvimento do bebê e durante este contato é possível suavizar os sentimentos negativos e promover uma relação positiva com a criança por meio da troca de energia com a mãe e com a equipe de enfermagem, essa troca simultânea contempla os componentes centrais da Ciência dos Seres Humanos Unitários de Rogers. Considerações finais: Esta reflexão contribuiu para a melhor compreensão do conhecimento empírico sobre: o toque, os campos de energia, o bebê, a mãe, a equipe de enfermagem, a teoria de Rogers, que juntamente ao referencial teórico, evidencia o quanto o tema é importante, atual e por intermédio dele novos conceitos podem ser desenvolvidos.
\end{abstract}

Palavras-chave: Neonatologia; Enfermagem holística; Modelos de enfermagem; Método canguru; Toque terapêutico.

\begin{abstract}
Objective: To promote reflections on comprehensive nursing care from the perspective of Martha Elizabeth Rogers Theory and the contact of babies with their mothers and the nursing staff. Methodology: This is an article of theoreticalreflective analysis supported by the theoretical framework of the theory of Martha Elizabeth Rogers, for which a search was carried out for productions that dealt with the topic in Academic Google and Pubmed, based on the experience of two experienced nurses in neonatal care, which occurred in June and July 2021. Results: In care practice in neonatology, touch benefits the baby's development and during this contact it is possible to soften the negative feelings and promote a positive relationship with the child through the exchange of energy with the mother and the nursing team, this way two-way approach contemplates the core components of Rogers' Science of Unitary Human Beings. Final considerations: This reflection contributed to a better understanding of the empirical knowledge about: touch, energy
\end{abstract}


fields, the baby, the mother, the nursing team, Rogers' theory, which together with the theoretical framework, shows how important the topic is, current and through it new concepts can be developed.

Keywords: Neonatology; Holistic nursing; Models, nursing; Kangaroo-mother care method; Therapeutic touch.

\section{Resumen}

Objetivo: Promover reflexiones sobre la atención integral de enfermería desde la perspectiva de la Teoría de Martha Elizabeth Rogers y el contacto de los bebés con sus madres y el personal de enfermería. Metodología: Se trata de un artículo de análisis teórico-reflexivo sustentado en el marco teórico de la teoría de Martha Elizabeth Rogers, para el cual se realizó una búsqueda de producciones que abordaran el tema en Google Académico y Pubmed, a partir de la experiencia de dos enfermeras experimentadas. en atención neonatal, que ocurrió en junio y julio de 2021. Resultados: En la práctica asistencial en neonatología, el tacto beneficia el desarrollo del bebé y durante este contacto es posible suavizar los sentimientos negativos y promover una relación positiva con el niño a través del intercambio de energía con la madre y el equipo de enfermería, de esta manera dos- Este enfoque contempla los componentes centrales de la ciencia de los seres humanos unitarios de Rogers. Consideraciones finales: Esta reflexión contribuyó a una mejor comprensión del conocimiento empírico sobre: el tacto, los campos energéticos, el bebé, la madre, el equipo de enfermería, la teoría de Rogers, que junto con el marco teórico, muestra la importancia del tema, actual y a través de él se pueden desarrollar nuevos conceptos.

Palabras clave: Neonatología; Enfermería holística; Modelos de Enfermería; Método madre-canguro; Tacto terapéutico.

\section{Introdução}

Martha Rogers, enfermeira visionária nascida em 1914, criou um sistema conceitual inovador e que, apesar do tempo transcorrido, continua a impactar a enfermagem até os dias atuais. Ela descreve o ser humano como um todo unificado, que tem sua própria integridade e mostra uma série de características que difere da soma de suas partes (Ali, 2017).

Rogers defendia que o ensino superior deveria guiar a prática profissional de enfermagem, quebrando os limites impostos, impulsionando a mudança da visão de mundo dos profissionais, tornando-os receptivos a novas ideias e práticas e acima de tudo, aptos a realizar pesquisas científicas para fortalecer a base teórica da enfermagem (Phillips, 2019).

Em sua teoria Martha Rogers aborda como pressupostos que os campos de energia humana e ambiental estão integrados, seus padrões e conceitos de bem-estar estão interrelacionados, a saúde está associada ao bem-estar e às alterações dos padrões (Mcewen \& Wills ,2016). Descreve também o enfermeiro como um ser criativo, com pontecial inovador que contribui para o enfrentamento das alterações dos padrões energéticos dos campos humano e ambiental. Desta maneira, ela permite ao enfermeiro explorar fenômenos que eram considerados paranormais, tornando-os métodos terapêuticos praticáveis, não invasivos e embasados cientificamente (Sá, 1994).

Ao compreender que o processo de interação entre os componentes centrais da teoria de Rogers representa uma percepção da integralidade dos seres humanos, do universo, do espírito energético, culminando em uma alma única que está em constante mudança e interação, o enfermeiro consegue identificar e experimentar essas manifestações por meio da presença integral e da consciência pandimensional (Phillips, 2015).

Rogers (1992) declara que: "Tendências holísticas abrem novas formas de pensar e soletram novas visões de mundo". Pela perspectiva holística as doenças são fruto do desequilíbrio do ser com o cosmos e com a natureza como um todo. Desta forma, por intermédio delas pretende-se reestabelecer a harmonia cósmica centrada no indivíduo (Stern e Guerriero, 2020). O ser humano holístico é constituído por um espectro de sistemas de energias interativas e vários fatores podem vulnerabilizá-lo à doença (Silva, Lima \& Bastos, 2015).

Contemplando o cuidado holístico destacam-se as práticas integrativas, que são técnicas que englobam: terapias físicas; hidroterapia; fitoterapia; nutrição; ondas, radiações e vibrações; terapia de exercícios individuais e terapias mentais e espirituais. Dentre as terapias mentais e espirituais estão inclusos o Reiki, a meditação, o toque terapêutico, o relaxamento psicomuscular, a cromoterapia, entre outras (Spezzia \& Spezzia, 2018). 
Sendo o profissional enfermeiro formado nos princípios holísticos, além de ser um dos profissionais que estabelece vínculos mais profundos, a enfermagem busca atender às necessidades dos indivíduos, percebendo-os como seres singulares e plurais (Siewert, et al., 2017).

O contato pele a pele para promoção do bem-estar do recém-nascido $(\mathrm{RN})$ foi preconizado com a Norma de Orientação para a Implantação do Método Canguru, publicada no Diário Oficial como Portaria GM nº 693, em 5 de julho de 2000, posteriormente revisada como Portaria n ${ }^{\circ}$ 1.683, de 12 de julho de 2007. O Método promove a participação dos pais e da família nos cuidados neonatais e estimula o contato pele a pele, de forma precoce e crescente desde o toque evoluindo até a posição canguru (Brasil, 2017).

Conforme uma revisão sistemática feita por Narciso et al. (2021), foram encontradas evidências de que o Método Canguru está relacionado a uma redução no tempo de hospitalização de bebês prematuros. Lee et al. (2021), em um estudo pioneiro, demonstraram que o contato pele a pele estabilizou e melhorou a função respiratória de prematuros em ventilação mecânica, com menores valores de volume corrente e pressão. Já em outra revisão sistemática, realizada por Pados e Hess (2020), evidenciaram que diversos estudos revelaram redução dos níveis de cortisol e aumento de ocitocina em bebês prematuros.

Na prática assistencial em neonatologia, percebe-se que o contato pele a pele, por meio da troca de energia, beneficia o desenvolvimento do bebê e durante o contato é possível suavizar os sentimentos negativos e promover uma relação positiva com a criança. Frente a esse cenário, o estudo teve por objetivo refletir sobre a relação existente entre a teoria de Martha E. Rogers e o contato pele a pele de bebês com suas mães e a equipe de enfermagem.

\section{Metodologia}

Trata-se de um estudo descritivo, que ocorreu nos meses de junho e julho de 2021, de análise teórico-reflexiva, elaborado a partir da vivência de duas enfermeiras que atuam na assistência neonatal em um Hospital Universitário, sobre a interrelação do contato pele a pele em neonatologia e a teoria de Martha Rogers, durante os cuidados prestados pela mãe e pela equipe de enfermagem.

Para subsidiar a reflexão, foi realizada uma busca por artigos que versassem sobre o tema, publicados na plataforma Google Acadêmico e no Pubmed, a partir dos descritores Martha Rogers, Ciência dos Seres Humanos Unitários, Pele a pele, Método Canguru, nos idiomas: português e inglês, utilizando o operador boleano AND.

Como critérios de inclusão, foram selecionados os textos que se enquadravam no objetivo da pesquisa, em qualquer idioma e disponibilizados para o leitor sob a forma de texto gratuito e completo. Foram excluídos os textos em duplicata.

Simultaneamente este estudo associa aspectos observados pelas autoras em seus ambientes de trabalho, nas unidades neonatais do Hospital Universitário, a saber: UTIN (Unidade de Terapia Intensiva Neonatal) e UCIN (Unidade de Cuidados Intermediários Neonatais) e a relação destes aspectos com a teoria de enfermagem de Martha E. Rogers.

Com base no estudo da teoria de Martha Rogers, e demais textos, a reflexão foi agrupada em três eixos:

- Conceitos-base para a compreensão da teoria dos seres humanos unitários de Martha Rogers;

- Campos energéticos, bem-estar e saúde de neonatos: considerações aplicadas ao cotidiano de cuidados de enfermagem;

- Relações entre o toque e a integralidade do cuidado: interação humana-ambiental e a prática de enfermagem.

\section{Resultados e Discussão}

\section{Conceitos-base para a compreensão da Ciência dos Seres Humanos Unitários de Martha Rogers}

Para desenvolver sua teoria de enfermagem, Rogers se baseou na diversidade de conhecimento de inúmeras fontes. Em 1970, publicou An Introduction to the Theoretical Basis of Nursing, no qual descreve a enfermagem como empírica e 
humanística, sempre preocupada com o bem-estar humano e a fornecimento de serviços qualificados plurais. Sua teoria dos seres humanos unitários estimula a promoção imaginativa e criativa do bem-estar de todos os seres humanos (Siewert, et al., 2017).

Dentro dos conceitos básicos em sua teoria encontramos a homeodinâmica, que corresponde às formulações teóricas que propiciam formas de descrever, esclarecer e antever diversos eventos relevantes para a prática profissional de enfermagem (Siewert, et al., 2017). A helicidade, ressonância e integralidade são princípios que estão interligados à homeodinâmica de tal forma que o cuidar se transforma em participar do processo de mudança das pessoas (Koffi; Fawcett, 2016).

Esses princípios se entrelaçam nas alterações nos padrões de energias com baixa e alta intensidade, dentro de uma onda contínua entre ambiente e ser humano (Dykeman, Loukissa, 1993). A pandimensionalidade permite perceber os fenômenos visíveis e invisíveis do campo energético para viver de maneira transcendental (Philips, 2015).

Dentro do pressuposto dos campos energéticos presente na teoria descreve que seres humanos e ambiente possuem energias metafísicas que geram equilíbrio produzindo boa saúde, e o desequilíbrio resulta em doença. Desta maneira o toque possibilita a harmonização, melhora no fluxo energético, por intermédio das mãos, estimulando a capacidade natural do corpo de curar a si mesmo (Garrett \& Riou, 2021).

\section{Campos energéticos, bem-estar e saúde de neonatos: considerações aplicadas ao cotidiano de cuidados de enfermagem}

Ao iniciar o percurso de internação de um RN em UTIN ou UCIN o enfermeiro e sua equipe percebem que durante os primeiros momentos depois do nascimento, os pais precisam de um tempo para adaptação à nova realidade, uma vez que a internação não foi o que idealizaram. A ordem correta seria após o parto, levar o bebê para casa, cuidar e suprir todas as suas necessidades.

Quando um RN é admitido na UTIN geralmente está acompanhado apenas pela equipe de enfermagem e pela equipe médica, o primeiro toque ao retirá-lo da incubadora de transporte é feito pelo enfermeiro, que o faz com cuidado e destreza para que o emaranhado de fios e tubos, que viabilizam os elementos necessários para manutenção da vida, não se desconectem e, concomitante a isso, observa o toque das mãos de quem está a ofertar o oxigênio ao RN, visto que este bebê possui um pulmão sensível e naquele momento crucial precisa de ajuda para respirar com auxílio da Ventilação por Pressão Positiva (VPP) com balão autoinflável, nesse ato o profissional responsável deve seguir com toques leves e precisos para que não ocorra o barotrauma ou volutrauma (Auten et al., 2001), permitindo desta maneira que ele possa sentir-se seguro e aconchegado como se ainda estivesse no útero materno.

O estresse relacionado ao cuidado, à submissão a eles pode resultar em alterações nos sistemas neurobiológicos, fisiológicos e hormonais do bebê. A presença do profissional não substitui a dos pais, entretanto, neste momento crítico, o RN deve receber o cuidado mais gentil e confortante possível, visando o alívio da dor e o controle do estresse, priorizando sua saúde e bem-estar, até que a mãe ou pai possam finalmente vê-lo (Peres, et al, 2021).

A avaliação física minuciosa do RN internado na UTIN e UCIN ocorre a fim de se obter subsídios para a definição de diagnósticos de enfermagem, planejamento e implementação de um plano de cuidados individualizado, tal avaliação é realizada minimamente, uma vez a cada vinte e quatro horas. Sobre a avaliação física propriamente dita, destacam-se os momentos de toque na relação enfermeiro-paciente: inspeção, palpação e ausculta; averiguar com atenção ao controle térmico durante o exame, cuidando para que o ambiente, as mãos e os instrumentos estejam em temperatura adequada, pois o RN tem pouca capacidade de tolerar alterações térmicas ambientais, não devendo ser exposto nem ao frio nem às temperaturas elevadas (Tamez,2013). Durante o exame físico, o enfermeiro também pode receber o toque das pequeninas mãos do seu cliente ou até mesmo um chute, devido aos movimentos ainda desorganizados do RN, o contato cada vez maior provoca estreitamento de laços entre profissionais e pacientes. 
O enfermeiro realiza diversos procedimentos técnicos, a exemplo de uma realiza uma punção venosa ou ao posicionar o RN para a aspiração de secreções no tubo orotraqueal e/ou vias aéreas, esse é um tipo de toque frequente e técnico, que o bebê admitido precisará conviver nos dias ou meses de internação na UTIN. Para manuseio do RN, o enfermeiro aplica cuidados com foco na segurança, melhora do suporte ventilatório, manuseio mínimo, minimização dos estímulos sensoriais e ambientais, contenção em ninhos para redução do estresse, almejando a manutenção em diminuir o desconforto e dor gerados. As intervenções de enfermagem são pautadas em estudos científicos por meio dos protocolos validados e revisados pelos enfermeiros assistenciais que atuam no serviço (Peres, et al, 2021).

A partir do momento que o bebê apresenta melhora em seu quadro clínico, ele passa a ter um contato contínuo com o toque da mãe dentro da UCIN. Nesta etapa além de ser submetido aos procedimentos técnicos, ele desfruta do toque afetivo, que aumenta seu campo energético durante procedimentos simples como banho, troca de fraldas, massagens, fisioterapia. Esse toque facilita a comunicação, criando vínculos entre o RN hospitalizado, a equipe de enfermagem e os pais, além de permear um cuidado humanizado (Ferreira \& Callado, 2013).

A equipe de enfermagem em unidade neonatal age favorecendo, além do contato entre o neonato e seus pais, a troca de energia entre eles. Assim como em outras áreas da enfermagem, ao mesmo tempo que lida com respostas e problemas de saúde, também trabalha com os processos da vida entre indivíduos, família, grupos e comunidades. Essas respostas são a preocupação central dos cuidados de enfermagem e para uma intervenção efetiva, o diagnóstico de enfermagem pode ser focado em problemas, riscos potenciais ou até mesmo a promoção de saúde.

A partir de 1994, foi incluído nos Diagnósticos de Enfermagem da North American Nursing Diagnosis Association (NANDA), o item Distúrbio no Campo Energético, que na descrição assemelha-se ao conceito de Martha Rogers, apresentando esse diagnóstico como a ruptura no fluxo vital de energia humana, que costuma ser um todo contínuo único, dinâmico, criativo e não linear. Aplicando NANDA-I na realidade da unidade neonatal, enquadram-se as seguintes características definidoras com os fatores relacionados (Herdman, 2018).

- Déficit de energia do fluxo energético relacionado à separação da mãe e do neonato devido à internação;

- Padrões fortes do campo de energia relacionados à implementação do toque da mãe ao seu RN;

- Padrões congestionados do campo de energia relacionados aos dispositivos tecnológicos de monitoramento instalados no neonato;

- Ritmos dissonantes dos padrões do campo de energia relacionados aos dispositivos sonoros presentes no ambiente que podem provocar estresse excessivo no RN, nos pais e na própria equipe de enfermagem.

Baseado na Teoria de Rogers, referenciando a interação dos campos energéticos humanos e ambiental, as modificações em um desses campos gera alteração no outro. Seguindo essa linha de raciocínio, o contato pele a pele proporciona a troca de energias entre os campos envolvidos, reorganizando o fluxo de energia vital desequilibrado, estimulando sua recuperação (Fernandes \& Nagay, 2002).

São descritas na literatura várias terapias associadas ao toque ou não toque, abordando o campo de energia. Dentre as terapias sem toque encontramos o Toque Terapêutico (TT) e as terapias com toque, que incluem tratamentos como o método canguru e massagem. Os cuidados envolvidos no método canguru, que envolve o contato pele a pele, no qual o RN é colocado diretamente no peito da mãe ou do pai, demonstram que o simples contato melhora as respostas simpático-vagais, evidenciado pela alteração da frequência cardíaca em prematuros (Radziewicz et al., 2018).

Associado ao estímulo do contato pele a pele, instituíram-se intervenções no sistema sensorial do RN hospitalizado, tais como: minimizar o excesso de luminosidade, ruídos, manipulação e a ampliação do tempo de permanência do pai ou da mãe nas 
24 horas para acompanhar o bebê, todas essas medidas unidas para promover o alívio do desconforto e da dor relacionados ao processo terapêutico, como forma de minimizar o estresse vivido pelo RN durante o período de internação.

Em uma revisão integrativa realizada por Furriel, Silva, Jennings e Christoffel (2020), evidenciou-se que medidas não farmacológicas de alívio da dor: sucção não nutritiva com sacarose $24 \%$ ou glicose $25 \%$, amamentação, posição canguru, cheiro da mãe, proporcionam resultados benéficos para redução de dor nos neonatos.

De acordo com os pressupostos da teoria de Martha Rogers, existe um campo energético humano em troca mútua e ininterrupta com o universo. Essas permutas contínuas resultam em trocas energéticas com variadas frequências de onda, intensidade e velocidade de resposta, representando a ressonância, componente da Ciência dos Seres Humanos Unitários. Ao seguir esta linha de raciocínio, o contato pele a pele, proporciona a troca de energias entre os campos envolvidos, reorganiza o fluxo de energia vital desequilibrado e estimula sua recuperação (Fernandes \& Nagay, 2002). Consequentemente, todas essas trocas geram melhora da saúde e bem-estar do RN, da família e da equipe envolvida.

\section{Relações entre o toque e a integralidade do cuidado: interação humana-ambiental e a prática de enfermagem}

Conforme Rogers, o indivíduo é um todo unificado, indivisível e totalmente integrado ao ambiente e deve ser apreciado com um olhar holístico. A prática de enfermagem fundamentada nesses conceitos, é capaz de implementar ações voltadas ao ser humano, em que os campos de energia humana e ambiental fluem continuamente entre eles, como uma onda ininterrupta que interage com as diversas dimensões do universo, sem uma simples redução a sistemas, órgãos e células (universo pandimensional) (Rogers,1992).

Na prática assistencial, o enfermeiro percebe o receio que pais e mães denotam em tocar ou falar com o bebê. Nesse interim, a equipe de enfermagem por estar mais próxima e cuidar continuamente do RN e por interagir maior tempo com a mãe, pai e familiares, precisa ser empática e refletir como é excruciante ver o seu RN envolvido por fios, conectado a diversos aparelhos, cercado por inauditos paramentados com suas toucas, máscaras, aventais, luvas, além de se comunicar com expressões que não fazem sentido para eles.

Pautada na teoria de Rogers, a enfermagem, uma ciência humanística e humanitária, envolvida em descrever a sinergia do ser humano com o todo, dentro dos princípios da integralidade, ressonância e helicidade, os campos de energia permanecem em constante troca por meio de sistemas abertos, nos quais ser humano e meio ambiente são indivisíveis. Desta maneira, o toque reflete a inter-relação entre eles, uma vez que existe uma troca dinâmica entre os padrões de energia. O toque pode ser contextualizado como um padrão de frequências vibracionais, assim como o bebê, em que cada um é um padrão único de frequências de energia (Gnatta; Kurebayashi; Turrini; Silva, 2016).

Ao considerar o significado literal de tocar, é perceptível que essa ação está essencialmente ligada ao cuidado prestado pela enfermagem. Durante a ação diária da equipe de enfermagem, o toque é utilizado tanto para execução de procedimentos quanto para acalento e aproximação do profissional com o paciente. Essa interação desenvolve uma relação que transcende os procedimentos técnicos (Dias, et al., 2008). A integração constante, indivisível e total dos campos energéticos humano-ambiental é designada de integralidade por Rogers (Sá, 1994).

Mediante o toque expressivo, verdadeiro e sincero, a enfermagem pode transmitir cuidado e apoio ao RN e à sua família, além de transmitir compreensão, apoio, calor, preocupação e acalento, na tentativa de minimizar o estresse pelo qual os envolvidos (mãe, bebê, equipe de enfermagem) são submetidos. Ao compreender o potencial de um toque, o profissional aumenta significativamente o sucesso da assistência de enfermagem, além de humanizar o processo (Ferreira e Callado, 2013).

Conforme Rangel et al. (2020), é possível promover um cuidado integral por meio do toque, percebendo o ser humano como um sistema inter-relacionado e em contínua mudança, harmonizando toda esta interação pelo equilíbrio dos campos 
energéticos. Corroborando dados encontrados por Narciso et al. (2021), Lee et al. (2021), Pados e Hess (2020), nos quais todos constataram melhoras como controle térmico adequado, reduz o estresse e a dor do RN, além da melhora do padrão respiratório.

Diante das situações vivenciadas por intermédio do contato pele a pele, afirma-se que o enfermeiro utiliza de sua criatividade para conceber métodos de trabalho inovadores, para a promoção do conforto ao paciente (Sá, 1994) e um processo de mudança com sua participação ativa (Gnatta; Kurebayashi; Turrini; Silva, 2016). Sempre empenhando-se na presença dos padrões humanos evidenciando a integridade da pessoa, da família ou da comunidade, sendo protagonista da mudança revolucionária dos padrões humanos que predizem a transformação do ser humano (Koffi; Fawcett, 2016)

\section{Considerações Finais}

O contato pele a pele, o toque, a interação dos campos energéticos e a integralidade no cuidado de enfermagem permitem relatar que essa estratégia proporciona conforto ao RN e tranquilidade às mães, inserindo-as no cuidado efetivo do seu filho(a), sendo um poderoso método de humanização, pois, além de aliviar a dor e proporcionar conforto ao RN, favorece o estreitamento dos laços afetivos entre ele e seus familiares.

O cuidado de enfermagem, por passar a maior parte do tempo em contato como bebê, deve partir de uma visão holística e humanística, de maneira que sejam minimizados os efeitos negativos gerados pela hospitalização, tanto para o bebê quanto para sua família.

Sob a ótica de Rogers, percebe-se que o toque ou o contato pele a pele está relacionado ao processo de cura, no qual a padronização dos campos de energia, possibilitam a melhoria das condições humanas e do bem-estar.

Este trabalho corrobora o conhecimento empírico sobre: o toque, os campos de energia, o bebê, a mãe, a equipe de enfermagem, a teoria Rogers, que, juntamente ao referencial teórico, evidencia o quanto o tema é importante, atual e pelo qual novos conceitos podem ser desenvolvidos.

Espera-se que este relato instigue a reflexão da atuação da equipe de enfermagem em neonatologia, pois o cuidado revelado pela teoria de Martha Rogers, Ciência do Ser Humano Unitário, está presente em nosso cotidiano, por meio da contínua interação entre os profissionais, família, bebês e meio ambiente.

\section{Referências}

Ali A. A. (2017). Understanding the Work of Nurse Theorists: A Creative Beginning, Third Edition. Research and theory for nursing practice, 31(4), 402-404. https://doi.org/10.1891/1541-6577.31.4.402

Alp, F., \& Yucel, S. (2020). The Effect of Therapeutic Touch on the Comfort and Anxiety of Nursing Home Residents. Journal Of Religion And Health, 60(3), 2037-2050. 10.1007/s10943-020-01025-4

Auten, R. L., Vozzelli, M., \& Clark, R. H. (2001). Volutrauma. What is it, and how do we avoid it? Clinics in perinatology, 28(3), 505-515. https://doi.org/10.1016/s0095-5108(05)70103-2

Brasil (2017). Atenção Humanizada ao Recém-nascido de Baixo Peso - Método Canguru. (3a ed.).

Dykeman, M., \& Loukissa, D. (1993). The Science of Unitary Human Beings: An Integrative Review. Nursing Science Quarterly, 6(4), 179-188. $10.1177 / 089431849300600406$

Dias, A., Oliveira, L., Dias, D., \& Santana, M. (2008). O toque afetivo na visão do enfermeiro. Revista Brasileira De Enfermagem, 61(5), 603-607. 10.1590/s0034-71672008000500012

Fernandes, Angela Cristina Puzzi y nagay, Silvana Capelleti (2002). Therapeutic touch - nonverbal communication: use in worker's health clinic. In: BRAZILIAN NURSING COMMUNICATION SYMPOSIUM, 8. Proceedings online. Escola de Enfermagem de Ribeirão Preto - USP, <http://www.proceedings.scielo.br/scielo.php?script=sci_arttext\&pid=MSC0000000052002000200021\&lng=en\&nrm=abn>.

Ferreira, F. R., \& Callado, L. M. (2013). O afeto do toque: benefícios nos recém nascidos. Rev. Med. Saúde. 2(2):112-9

Furriel, C., Silva, G., Jennings, J., \& Christoffel, M. (2020). Medidas não farmacológicas para alívio da dor do recém-nascido a termo: revisão integrativa. Research, Society And Development, 9(9), e687997721. 10.33448/rsd-v9i9.7721 
Garrett, B., \& Riou, M. (2021). A rapid evidence assessment of recent therapeutic touch research. Nursing open, 10.1002/nop2.841. Advance online publication. https://doi.org/10.1002/nop2.841

Gnatta, J. R., Kurebayashi, L. F., Turrini, R. N., \& Silva, M. J. (2016). Aromaterapia e enfermagem: concepção histórico-teórica [Aromatherapy and nursing: historical and theoretical conception]. Revista da Escola de Enfermagem da U S P, 50(1), 130-136. https://doi.org/10.1590/S0080-623420160000100017

Herdman, T. Heather et al. (2018). Diagnósticos de enfermagem da NANDA-I: definições e classificação 2018-2020. Artmed.

Koffi, K., \& Fawcett, J. (2016). The Two Nursing Disciplinary Scientific Revolutions. Nursing Science Quarterly, 29(3), 247-250. 10.1177/0894318416648782

Lee, J., Parikka, V., Lehtonen, L., \& Soukka, H. (2021). Parent-infant skin-to-skin contact reduces the electrical activity of the diaphragm and stabilizes respiratory function in preterm infants. Pediatric research, 1-5. Advance online publication. https://doi.org/10.1038/s41390-021-01607-2

Mcewen, M., \& Wills, E. (2016). Bases teóricas para enfermagem. (4a ed.), Artmed.

Narciso, L., Beleza, L., \& Imoto, A. (2021). The effectiveness of Kangaroo Mother Care in hospitalization period of preterm and low birth weight infants: systematic review and meta-analysis. Jornal De Pediatria. 10.1016/j.jped.2021.06.004

Pados, B., \& Hess, F. (2020). Systematic Review of the Effects of Skin-to-Skin Care on Short-Term Physiologic Stress Outcomes in Preterm Infants in the Neonatal Intensive Care Unit. Advances In Neonatal Care, 20(1), 48-58. 10.1097/anc.0000000000000596

Peres, A. L. et al. (2021). Cuidados de enfermagem ao recém-nascido nos distintos cenários: revisão integrativa. Advances in Nursing and Health, 3, 31-47.

Phillips, J. (2014). Martha E. Rogers. Nursing Science Quarterly, 28(1), 42-48. 10.1177/0894318414558608

Phillips, J. (2015). Rogers' Science of Unitary Human Beings. Nursing Science Quarterly, 29(1), 38-46. 10.1177/0894318415615112

Phillips, J. (2019). Unitariology and the Changing Frontiers of the Science of Unitary Human Beings. Nursing Science Quarterly, 32(3), 207-213. $10.1177 / 0894318419845404$

Radziewicz, R., Wright-Esber, S., Zupancic, J., Gargiulo, D., \& Woodall, P. (2018). Safety of Reiki Therapy for Newborns at Risk for Neonatal Abstinence Syndrome. Holistic Nursing Practice, 32(2), 63-70. 10.1097/hnp.0000000000000251

Rangel, R., Medeiros, A., Oliveira, A., Rodrigues, S., Scarton, J., \& De Siqueira, H. (2020). Efeitos do toque terapêutico no cuidado integral ao ser humano à luz do Pensamento Ecossistêmico. Research, Society And Development, 9(4), e176942421. 10.33448/rsd-v9i4.2421

Sá, A. (1994). A ciência do ser humano unitario de Martha Rogers e sua visão sobre a criatividade na prática da enfermagem. Revista Da Escola De Enfermagem Da USP, 28(2), 171-176. 10.1590/0080-6234199402800200171

Siewert, J., Rodrigues, D., Malfussi, L., Andrade, S., \& Erdmann, A. (2017). Management of integral care in nursing: reflections under the perspective of complex thinking. REME: Revista Mineira De Enfermagem, 21. 10.5935/1415-2762.20170057.

Rogers M. E. (1992). Nursing science and the space age. Nursing science quarterly, 5(1), 27-34. https://doi.org/10.1177/089431849200500108

Spezzia, S., \& Spezzia, S. (2018). O uso do reiki na assistência à saúde e no sistema único de saúde. Revista De Saúde Pública Do Paraná, l(1), 108-115. $10.32811 / 2595-4482.2018 v 1 n 1.49$

Stelmak, A. P., Mazza, V. A., \& Freire, M. H. S. (2017). O valor atribuído pelos profissionais de enfermagem aos cuidados preconizados pelo método canguru. Rev enferm UFPE online, 11(9), 3376-85.

Stern, F. L., \& Guerriero, S. (2020). Terapias holísticas: uma análise do sistema médico na Nova Era. EDUC, 2020.

Tamez, R. N.; \& Silva, M. J. P. (2013) Enfermagem na UTI neonatal - Assistência ao recém-nascido de alto risco. (5a ed.), Guanabara Koogan. 